\title{
Wave-packet recollision and pulse-shape effects in high-harmonic generation
}

\author{
J. B. Watson, ${ }^{1}$ A. Sanpera, K. Burnett, ${ }^{1}$ and P. L. Knight ${ }^{2}$ \\ ${ }^{1}$ Clarendon Laboratory, Department of Physics, University of Oxford, Parks Road, Oxford OX1 3PU, United Kingdom \\ ${ }^{2}$ Blackett Laboratory, Optics Section, Imperial College, Prince Consort Road, London SW7 2BZ, United Kingdom
}

(Received 22 March 1996)

\begin{abstract}
The recollision model of harmonic generation in a time-varying field has two elements: the emission due to a recolliding wave packet, and the quasiperiodic emission of wave packets in successive cycles. By discussing these issues separately, we explain the origin of blueshifting, and the "smearing" of the harmonic spectrum. We find that such a model shares many of the qualitative features of the semiclassical theories of harmonic generation. [S1050-2947(97)09401-8]
\end{abstract}

PACS number(s): 42.50.Vk, 32.80.Rm, 42.65.Ky

\section{INTRODUCTION}

In this paper we will describe some of the critical features of high harmonic generation in a pulsed laser field. In particular we will consider the origin of the single-atom blueshift and loss of harmonic resolution that we have previously identified by solving the time-dependent Schrödinger equation [1]. Before doing this we will discuss the salient features of the emission in a constant amplitude field. The spectrum of harmonics in a constant amplitude field has the same characteristic behavior for a wide range of experimental parameters: a plateau of harmonics of approximately equal intensity followed by a sharp cutoff, at an energy given by $I_{p}+3.2 U_{p}$, where $I_{p}$ is the binding energy of the initial state, and $U_{p}$ is the ponderomotive energy which, for a laser of frequency $\omega_{0}$ and amplitude $\epsilon_{0}$, is given by $U_{p}$ $=\epsilon_{0}^{2} /\left(4 \omega_{0}^{2}\right)$ [2]. These features have been explained by means of a semi-classical model based on tunneling and recollision proposed by Corkum [3], and also by Kulander, Schafer, and Krause [4]. This is a three-step model, the first step of which is the promotion of an electron into the continuum, which is thought of as occurring via tunneling. Once it has escaped, the electron will oscillate under the influence of the electric field, and, depending on the phase at which the electron is produced, will have some probability of returning to the nucleus. If the returning electron is recaptured by the nucleus, it may emit a single, high-energy photon. From a simple analysis of the possible classical trajectories of a free electron in the field, it is possible to obtain the cutoff law quoted above.

Lewenstein et al. [5] demonstrated that by making a strong-field approximation (i.e., assuming that the effect of the atomic potential on the continuum electrons can be neglected compared with the laser field) this recollision picture of the harmonic generation can be recovered from a purely quantum-mechanical treatment. In a recent paper Kan et al. [6] extended the Lewenstein model to describe the features of high harmonic generation in a pulsed field. Their model has led to a simple description of the single-atom blueshift in terms of the phase of the returning electrons.

In this paper we extend the model of the recollision process developed previously by Protopapas et al. [7] which describes the harmonic generation in terms of the spectrum due to a single encounter of a tunneling wave packet with the atomic core coupled with the periodic nature of the recollision. The exact mechanism of the single-collision spectrum depends on the details of the system. As is usual in the study of harmonic generation, we consider only the radiation due to the mean dipole, and neglect the dipole fluctuations $[8,9]$. In this limit, for an electron colliding with a completely depleted atomic core, then only continuum-continuum interactions contribute to the coherent spectrum, and therefore the response can be described in terms of bremsstrahlung, as discussed by Karapetyan and Fedorov [10]. For the more typical case where there is a large bound-state population, it is the dipole moment between the bound and continuum parts of the wave function that is important, as has been described by Corkum [11].

In this paper we shall contrast the effect of a single wave packet tunneling out of the atom and recolliding multiple times, with the effect of a series of different wave packets undergoing a single recollision. In order to treat this problem correctly, one must also consider the oscillating phase of the ground state. We will then extend this model to the case of time-dependent pulse shapes, and demonstrate that such a model shares many of the features of the recollision theory. Moreover, we shall see that quite general features of the spectrum can be understood in quantal as well as semiclassical terms.

\section{QUANTUM RECOMBINATION MODEL}

For much of this paper we will use the terminology of the semiclassical theories (i.e., tunneling, oscillation in the field, recollisions, etc.). We should emphasize, however, that our calculations and conclusions are not dependent on the validity of the semiclassical models, although the fact that our work agrees with their predictions is further justification for the insight they provide. In our discussion we will follow the approach of Protopapas et al. [7] in considering two distinct aspects of the harmonic generation, first the radiative emission due to the behavior of a single continuum wave packet, and second the effect of the periodicity of the driving field. In order to simplify our initial discussion, we will assume that the depletion of the ground state is small, and that the laser field has a constant amplitude. We shall, however, return to the effect of ground-state depletion at the end of this section. The important effects due to a time-varying field 
amplitude will be taken up in a later section. In the limit of no depletion, an identical continuum wave packet is produced every half-cycle, at the same phase relative to the driving field. Furthermore, the behavior of each wave packet in the field will be the same, and therefore one might expect a series of identical recombination events. This last assumption is only true in the limit where each wave packet only encounters the core once; if a given wave packet encounters the core more than once, the situation is more complicated. In this case it is convenient to still think of wave packets produced in different cycles as distinct, even though they overlap. We shall see later that these multiple collisions have a definite effect on the harmonic spectrum. For the moment we shall presume that the dominant features of the emission spectrum can be described assuming strict periodicity [7].

If each recollision event is essentially the same, the contribution made to the dipole acceleration by each will be identical, simply shifted in time by half of the laser period. In mathematical terms we can write the dipole acceleration (in the time domain) as the convolution of the dipole acceleration due to a single recollision, with a series of $\delta$ functions separated by $\tau / 2$, where $\tau=2 \pi / \omega_{0}$ is the laser period. If we consider two consecutive emissions and recombinations, one will be a mirror image of the other about the origin. Since the dipole acceleration operator has an odd parity, there will be a sign change between the two contributions, and the sign of our $\delta$ functions will therefore alternate. In order to obtain the frequency spectrum of the radiation, we have to take the Fourier transform of the dipole acceleration. From an application of the convolution theorem, the spectrum is then simply the product of the Fourier transforms of the two functions in the convolution. Since the Fourier transform of our series of $\delta$ functions is simply another set of $\delta$ functions with peaks at $(2 n+1) \omega_{0}$, the spectrum is the single-cycle spectrum, modulated by peaks at odd harmonics of the laser frequency (this is analogous to diffraction grating in optics [12]).

In the argument so far we have neglected changes in the ground-state population, whereas in any real harmonicgeneration experiment ionization is necessarily present. When the ground-state population decreases we need to modify our assumption that successive wave packets are identical. It is reasonable, however, to assume that each wave packet has a normalization proportional to the ground state population when it is produced, but that otherwise they are identical. Similarly the dipole induced when the wave packet recombines should be proportional to the ground-state amplitude at the "time" of recollision. We can add this depletion effect to our model by multiplying each of our $\delta$ functions in the time domain by a factor $g\left(t_{r}\right) g\left(t_{r}-\tau / 2\right)$, where $g(t)$ is the amplitude of the ground state. In other words we have to multiply our series of $\delta$ functions by some envelope function which in general is slowly varying. The effect of this can be obtained by another application of the convolution theorem. The harmonic peaks are broadened; however, since the envelope is slowly varying, this broadening is small.

In the next section we will describe our numerical method, but first we should clarify what we mean by the single-wave-packet spectrum. So far we have discussed harmonic generation in terms of a series of wave packets, each returning to the core once. We also assumed that, for a given energy, during a single recollision, there is only one possible trajectory. In reality, for a single wave packet, there are two possible trajectories which will generate harmonics in the plateau region. Furthermore, there is also a finite probability of each wave packet oscillating close to the core and recolliding more than once. The effect of each subsequent recollision will decrease due to dispersion of the wave packet, but nevertheless we still need to take this into account. These recollisions do not change the basic philosophy of our model. We can still define the spectrum as the convolution of the response due to a single wave packet with a series of $\delta$ functions. The difference is that in this case the single-wavepacket response will extend over several laser cycles. This presents a small problem in verifying our model by numerical calculation, since it is difficult to isolate the response due to a single wave packet. The solution to this problem is to consider the dipole acceleration in terms of the convolution of the set of $\delta$ functions with the half-cycle response. If we consider the dipole acceleration during half a cycle, at some time during a flat pulse there will be a contribution due to the first recollision of one of the wave packets, plus contributions due to trajectories with long return times and wave packets that have already crossed the core one or more times. While repeating a single half-cycle does not reproduce exactly the full dipole acceleration, we will show that it gives a reasonable approximation of the correct spectrum. This gives us a convenient method of examining periodic effects in the spectrum. In particular, it allows us to identify the effect of multiple trajectories, with the same kinetic energy, from a single wave packet. We shall see later that this point is critical to the details of the harmonic spectrum, particularly when using a pulsed field.

\section{NUMERICAL METHOD}

Our calculations of the harmonic response are based on numerical integration of the time-dependent Schrödinger equation (TDSE) in the length gauge using the CrankNicholson method [13]. We use a one-dimensional equation with the soft-core potential [14]

$$
V(x)=\frac{-Z}{\left(1+x^{2}\right)^{1 / 2}} .
$$

We choose $Z=2$ in order to model an ion, since previous studies have indicated that pulse-shape effects are more important for higher binding energies [1].

The effect of using a one-dimensional potential has been discussed elsewhere [15]. The principal argument against the use of one-dimensional models of recollision concerns the effect of transverse spreading of the continuum wave packet in three-dimensional calculations. The effect of this spreading is an important issue in its own right, and one that is beyond the scope of the current paper. The use of the onedimensional model for the present calculation can be justified by the fact that the pulse-shape effects discussed here are also observed in three-dimensional calculations [1].

In order to study the effects of the periodicity we make two different calculations of the harmonic spectrum due to any particular pulse shape. The first method is to calculate the dipole acceleration for the entire duration of the pulse. 
The second is to calculate the response to a single half-cycle, and then to build up the dipole acceleration from a set of half-cycle responses. In the case of a constant amplitude this amounts to taking a single half-cycle response $d_{s}$ and repeating it over the pulse [7],

$$
\ddot{d}(t)=\sum^{n}(-1)^{n} d_{s}(t-n \tau / 2) .
$$

For a time-dependent pulse we calculate the half-cycle response at different intensities, and use them to model the shape of the pulse.

\section{SINGLE-CYCLE HARMONIC SPECTRUM}

In this section we will consider the details of the singlecycle response. We will interpret the spectrum in terms of tunneling and recollision, although the periodic model above is independent of the exact mechanism of harmonic generation. We obtain the harmonic spectrum by calculating the expectation value of the dipole acceleration, i.e.,

$$
\ddot{d}(t)=\langle\Psi(x, t)|\ddot{d}| \Psi(x, t)\rangle .
$$

We can gain extra insight into the emission process by considering the projection of the dipole acceleration onto the ground state, i.e.,

$$
\ddot{d}_{p}(t)=\langle\Psi(x, t) \mid g\rangle\langle g|\ddot{d}| \Psi(x, t)\rangle .
$$

We start by writing the wave function in the form

$$
\Psi(x, t)=a(t) \exp \left(-i \omega_{g} t\right)|g\rangle+\Psi_{f}(x, t) .
$$

Here we separated the ground state $|g\rangle$ from the remainder of the wave function $\Psi_{f}$, and factored out its oscillating phase.

Substituting into Eq. (3), we find that the full spectrum is given by

$$
\begin{aligned}
\ddot{d}(t) & =a^{*}(t) \exp \left(i \omega_{g} t\right)\left\langle g|\ddot{d}| \Psi_{f}(x, t)\right\rangle+\left\langle\Psi_{f}(x, t)|\ddot{d}| \Psi_{f}(x, t)\right\rangle \\
& =\left\langle\Psi_{g}(x, t)|\ddot{d}| \Psi_{f}(x, t)\right\rangle+\left\langle\Psi_{f}(x, t)|\ddot{d}| \Psi_{f}(x, t)\right\rangle
\end{aligned}
$$

where $\Psi_{g}(x, t)=a(t) \exp \left(i \omega_{g} t\right)|g\rangle$. The first term in this equation corresponds to transitions from the excited and continuum states back to the initial state, while the second term is due to transitions that only involve excited and continuum states.

On the other hand, the projected spectrum is given by

$$
\begin{aligned}
\ddot{d}_{p}(t) & =a^{*}(t) \exp \left(i \omega_{g} t\right)\left\langle g|\ddot{d}| \Psi_{f}(x, t)\right\rangle \\
& =\left\langle\Psi_{g}(x, t)|\ddot{d}| \Psi_{f}(x, t)\right\rangle .
\end{aligned}
$$

Comparison of Eqs. (6) and (7) indicate that, by projecting the dipole acceleration onto the ground state, it is possible to isolate the response due to transitions back to the ground state.

In a previous paper Krause, Schafer, and Kulander [16] demonstrated that this projected spectrum was similar to the full dipole acceleration spectrum, except that the structure after the cutoff was lost. In our calculations we have found that, if the ionization is small, the spectra calculated by the two methods are almost identical. This being the case, the second term in Eq. (6) must be negligible. This analysis indicates that the dominant contribution to the harmonic spectrum comes from the oscillating dipole moment induced between the bound state $\left(\Psi_{g}\right)$ and the excited and continuum $\left(\Psi_{f}\right)$ parts of the wave function. Furthermore, since $\Psi_{g}$ is strongly localized at the core, it is only the part of the continuum wave function close to the core that contributes-i.e., the recolliding wave packet (and also, as we shall see later, the tunneling part). This reproduces the explanation of the emission process given by Corkum [11].

We will consider two separate contributions to the spectrum, the first of which can be thought of as due to the tunneling process. The idea of harmonics generation due to tunneling was first predicted by Brunel [17], and was later utilized by Rae and Burnett [18] in a self-consistent model of propagation effects. When the wave packet is first produced in the continuum, it is close to the origin, and so an oscillating dipole is induced between the bound state and the continuum; therefore the atom radiates. Since at this stage the wave packet has not been accelerated by the laser field, the (kinetic) energy associated with it is small, and therefore the spectrum falls off quickly with increasing energy. We can demonstrate this by calculating the atomic response to half of a laser cycle. The result is plotted in Fig. 1(a) (solid curve). In a laser pulse this tunneling event is repeated periodically, and therefore the spectrum contains peaks at the odd harmonics. We will demonstrate the effect of the periodicity below, but first we will consider a second contribution due to electrons returning to the core, and recombining with the ground state, i.e., the recollision in the semiclassical model. In the first half-cycle there is tunneling, but the wave packet does not return to the core until the second half-cycle (when the field switches direction). Each half-cycle, from the second onwards, contains both tunneling and recollision events. This can be demonstrated by looking at the response during second half-cycle of the pulse: the dashed curve in Fig. 1(a). One can see in the figure that for energies below $8 \omega_{0}$ the two spectra are essentially the same, from which we deduce that this is the region where the "tunneling" process dominates. Beyond this region the solid curve continues to decrease rapidly in intensity, while the dashed curve exhibits a clear plateau. We conclude that the presence of the plateau is due to the recollision of the first wave packet. As predicted by the semiclassical model the plateau has a cutoff at $I_{p}+3.2 U_{p}$. Figure 1(b) shows the response calculated over the first (solid curve) and second (dashed curve) cycles. We can see that in both cases there are harmonic peaks up to the ninth harmonic, while after this point there are only clear harmonic peaks in the second cycle response. This difference is a simple consequence of the periodicity. The odd harmonicsappear when a spectrum (or part of a spectrum) is repeated in time at intervals of $\tau / 2$ with alternating sign. In the first cycle two wave packets tunnel out from the core, but only a single wave packet recollides. Therefore the tunneling part of the spectrum contains peaks at the harmonics, but the recollision part does not. In the second cycle there are two wave packets created by tunneling and two recolliding wave packets (the first wave packet to collide during the second cycle 

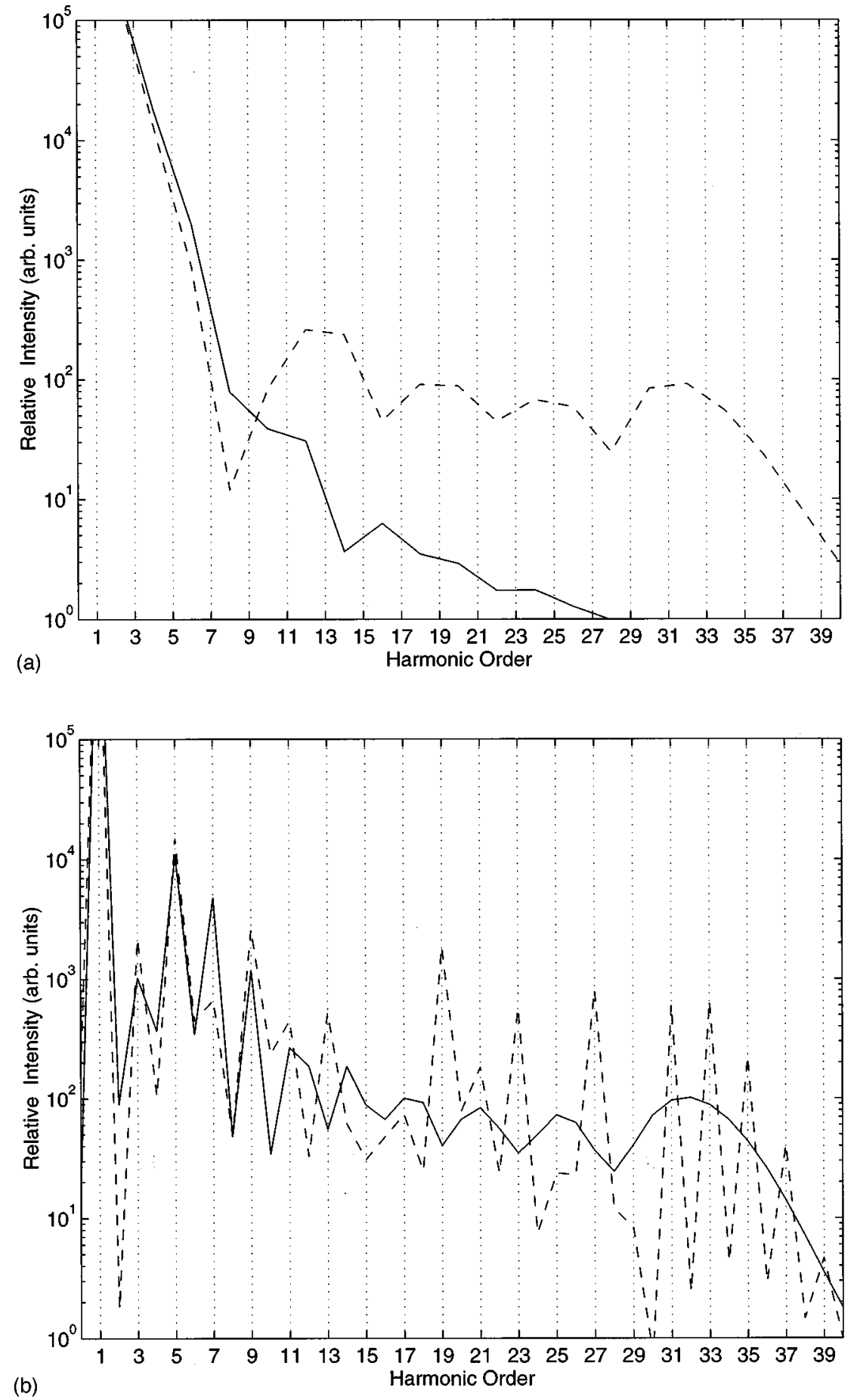

FIG. 1. (a) The spectrum generated during half a laser cycle in $\mathrm{He}^{+}$at intensity $1.5 \times 10^{15}$ $\mathrm{W} / \mathrm{cm}^{2}$ and wavelength $372 \mathrm{~nm}$, using the first half-cycle (solid curve) and the second half-cycle (dotted curve). (b) The spectrum generated during a single laser cycle in $\mathrm{He}^{+}$at intensity $1.5 \times 10^{15} \mathrm{~W} / \mathrm{cm}^{2}$ and wavelength $372 \mathrm{~nm}$, using the first cycle (solid curve) and the second cycle (dotted curve). was produced during the first cycle) and therefore the harmonics appear across the whole spectrum. We note that in the second-cycle spectrum certain harmonics are suppressed. We will show in a later section that this is due to recollisions at different phases (relative to the driving field) from a single wave packet.

Before concluding this section we will give further evidence of the recollision model. In Fig. 2 we plotted the electron probability density as a function of position and time as a colorscale plot for the first two cycles of a flat pulse. In this plot the ground-state population has been removed in order to emphasize the tunneling part. The first wave packet is emitted over the first part of the cycle, and recollides within one cycle. If we take the second half of this cycle then the only recollision is from a single wave packet. If, however, we were to consider the next half-cycle we would receive contributions due to both the first wave packet returning for the second time and the next wave packet returning for the first time. As already pointed out by Protopapas et al. [7], the interference between these different wave packets will lead to extra structure in the wave function; this explains the origin of the fringes in the second cycle plotted in Fig. 2. We will show later that these recollisions make an important contribution to the spectrum. 


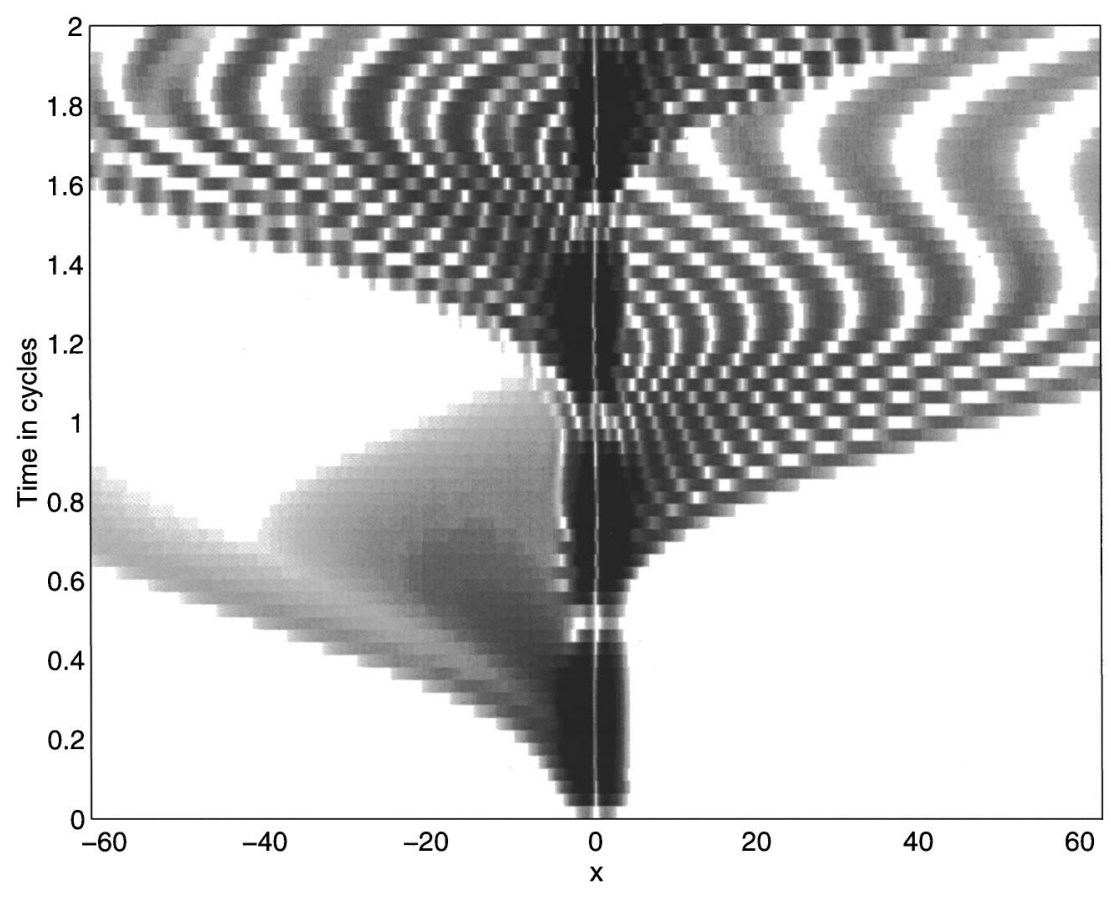

FIG. 2. A contour plot of the probability density as a function of $x$ and $t$ for a two-cycle pulse in $\mathrm{He}^{+}$at intensity $1.5 \times 10^{15} \mathrm{~W} / \mathrm{cm}^{2}$ and wavelength $372 \mathrm{~nm}$.

\section{HARMONIC GENERATION WITH A CONSTANT INTENSITY}

In previous sections we emphasized the importance of the periodicity of the wave-packet production in generating harmonics. In this section we will verify the periodic nature of the harmonic generation by comparing the response to a full pulse, with the spectra obtained by periodically repeating the single half-cycle response. This approach will also allow us to distinguish between the response due to the first recollision, and effects due to further recollisions of the same wave packet. We calculated the harmonic spectrum for $\mathrm{He}^{+}$using an eight-cycle flat pulse of intensity $1.5 \times 10^{15} \mathrm{~W} / \mathrm{cm}^{2}$ at wavelength $372 \mathrm{~nm}$. In Fig. 3(a) we plot the harmonic spectrum obtained by solving the TDSE for the full eight cycles. The result is the familiar plateau of harmonics with a sharp cutoff at a position given by $I_{p}+3.2 U_{p}$. Figure $3(\mathrm{~b})$ shows the same spectrum calculated by integrating the Schrödinger equation for the first cycle only, and repeating the dipole acceleration due to the second half of this cycle, with alternating sign to produce the eight-cycle response. (For comparison we have included the full spectrum as a dotted curve.) It is clear that the cutoff in Fig. 3(b) is correct, as are the positions of the harmonics. There are, however, a couple of clear differences between Figs. 3(a) and 3(b). First, although the plateau is correct in the single-cycle model, there are clear harmonics after the cutoff, whereas in Fig. 3(a) there is still a background but with no clear peaks. The origin of these extra harmonics is that in repeating a single halfcycle we have made the background noise in the spectrum periodic.

The second point concerns the intensity of the plateau harmonics. In Fig. 3(a) there is a large variation in the harmonic intensities, with some harmonics (namely, the 17th and 29th) suppressed, whereas in the single-cycle model the peaks are of approximately constant intensity. By choosing the second half-cycle of the pulse we have neglected a couple of critical contributions to the harmonic spectrum; we have only included the first recollision of each wave packet, and we have cut off part of the recollision of this wave packet. Simulations, both classical, and using the strong-field approximation (see, for example, Lewenstein et al. [5]) have shown that for a given return energy (outside the cutoff region) there are two possible trajectories. For a $\sin \left(\omega_{0} t\right)$ field the highest-energy trajectory occurs at a phase $1.86 \pi$, and for energies below the maximum there are two possible classical trajectories-one before and one after the maximum. This difference between the cutoff and plateau harmonics has also been demonstrated in solutions of the time-dependent Schrödinger equation by Maquet et al. [19]. By using a wavelet analysis of the dipole acceleration, they concluded that the cutoff harmonics are produced only once in each half-cycle, while the plateau harmonics are produced at several different times. Here we will make no detailed analysis of the temporal dependence of various parts of the spectrum. We can, however split the single-wave-packet response into two parts; contributions that occur in the same cycle as the tunneling event, and contributions that occur in subsequent cycles. By sampling different cycles within a flat pulse, we can differentiate between these two contributions.

If, instead of sampling the second half-cycle, we take the fourth half-cycle, there will be contributions due to the tunneling of a new wave packet and the recombination of the previous one, as before. There will also be contributions due to trajectories with a long return time, and recollisions of older wave packets. The spectrum obtained by sampling the second cycle is plotted in Fig. 3(c); again we show the result of the full calculation as a dotted curve. In this case we still find the plateau with harmonic peaks, but there is strong modulation of the peaks. In fact the spectrum bears a striking similarity to that obtained for the full eight-cycle pulse. From this we conclude that the modulation of the harmonic peaks is due to multiple contributions to a given part of the spectrum by a single wave packet.

We have therefore confirmed that the harmonics arise due to the periodicity of the electron recollisions (Protopapas et al. [7]), and demonstrated the validity of writing the har- 


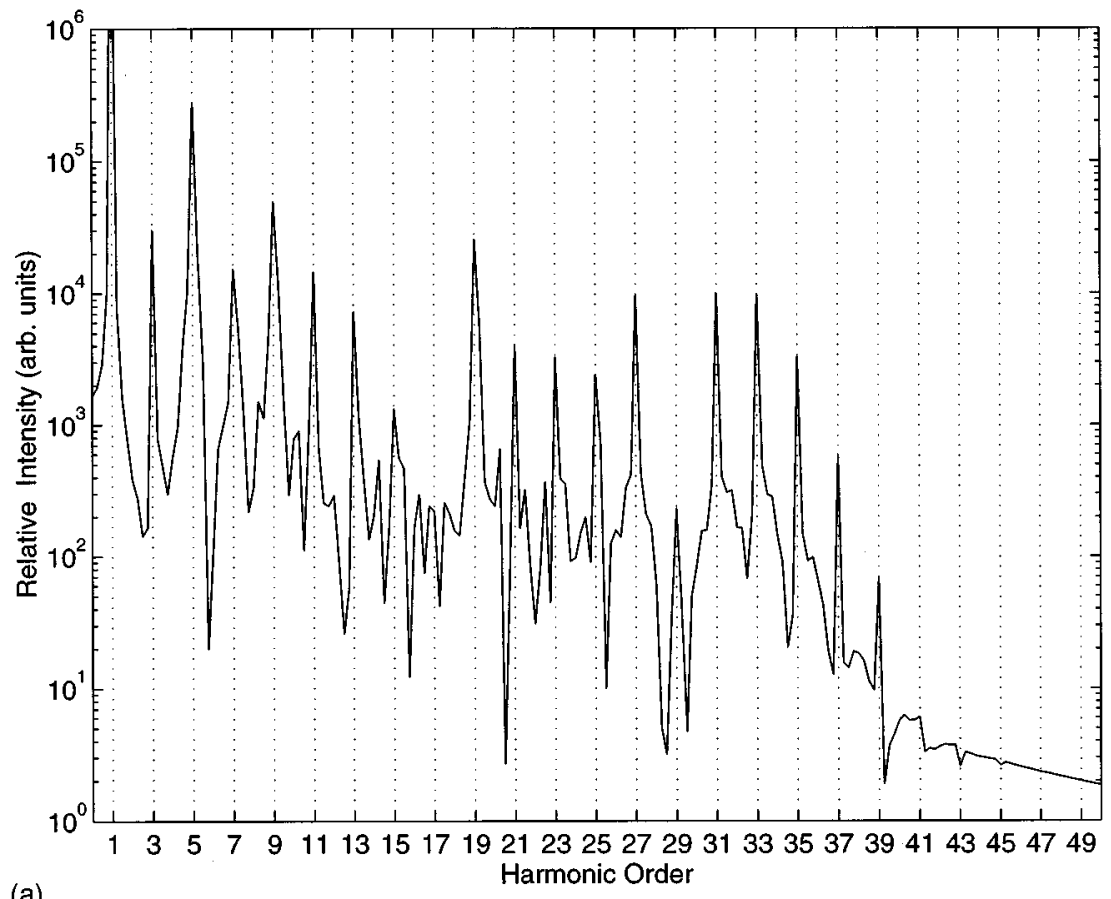

(a)

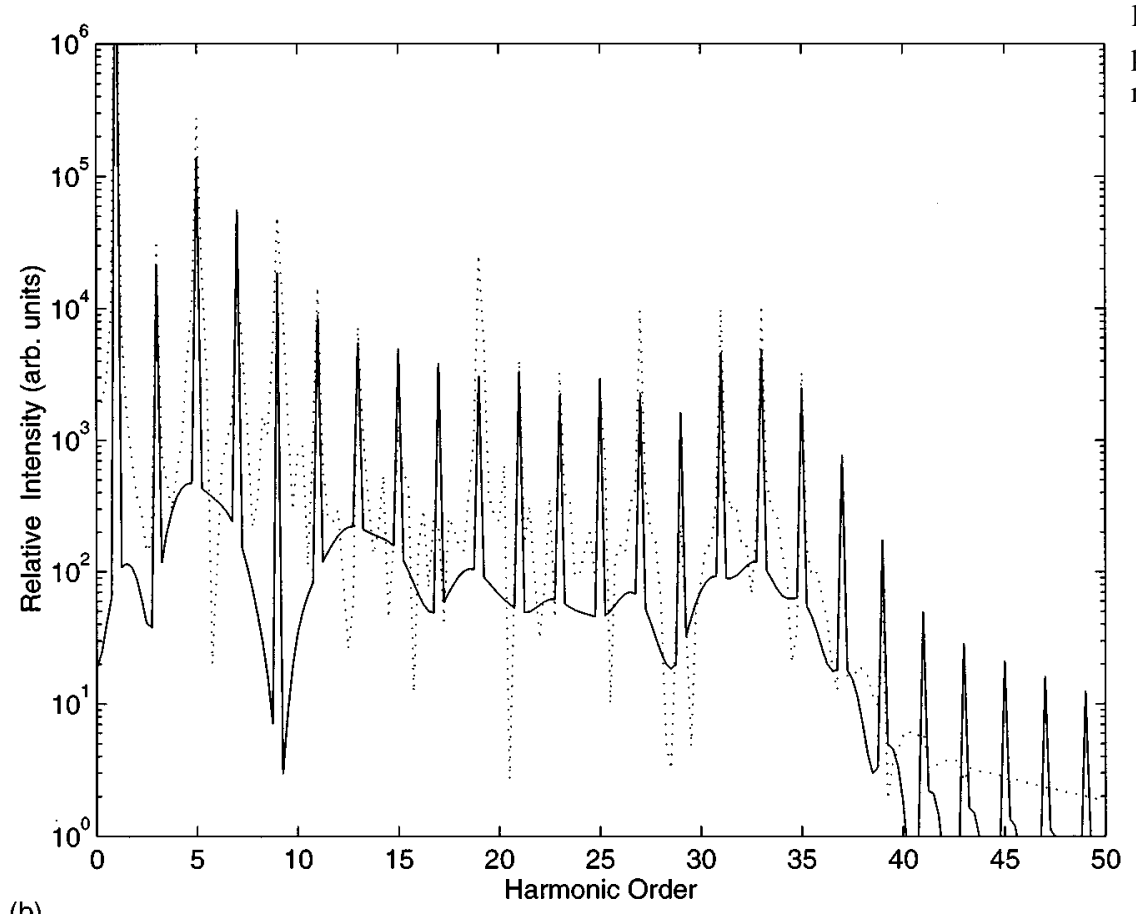

FIG. 3. The harmonic spectrum generated from $\mathrm{He}^{+}$using an eight-cycle pulse of intensity $1.5 \times 10^{15} \mathrm{~W} / \mathrm{cm}^{2}$ and wavelength $372 \mathrm{~nm}$ calculated by (a) using a full eight-cycle pulse, (b) periodically repeating the first cycle, and (c) periodically repeating the second cycle.

(b)

monic spectrum as a convolution of the response due to a single-wave packet with a set of periodic $\delta$ functions. The case where we sampled the second half-cycle is equivalent to considering only the first cycle of the single-wave-packet response, while sampling the fourth half-cycle is equivalent to extending the calculation of the single-wave-packet response to two laser periods. In the latter case the contribution due to a single wave packet will show extra structure, not necessarily harmonic peaks, and therefore there is a modulation of the harmonic efficiency.

So far we have developed the idea of harmonic generation due to a series of identical wave packets, created at intervals of half the laser period, recolliding with the nucleus. In the remainder of this paper we shall demonstrate that, with a simple modification, our model can be used to describe the response to a pulse with a time-dependent intensity.

\section{HARMONIC GENERATION USING A TIME-DEPENDENT PULSE}

In a previous paper [1] we demonstrated that when the laser intensity increases during the pulse, there is a blueshift of the harmonics. Furthermore, when there is a rapid time dependence, the structure of the harmonics may become "smeared out," so that it is no longer possible to distinguish individual peaks. To illustrate these results we calculated the 


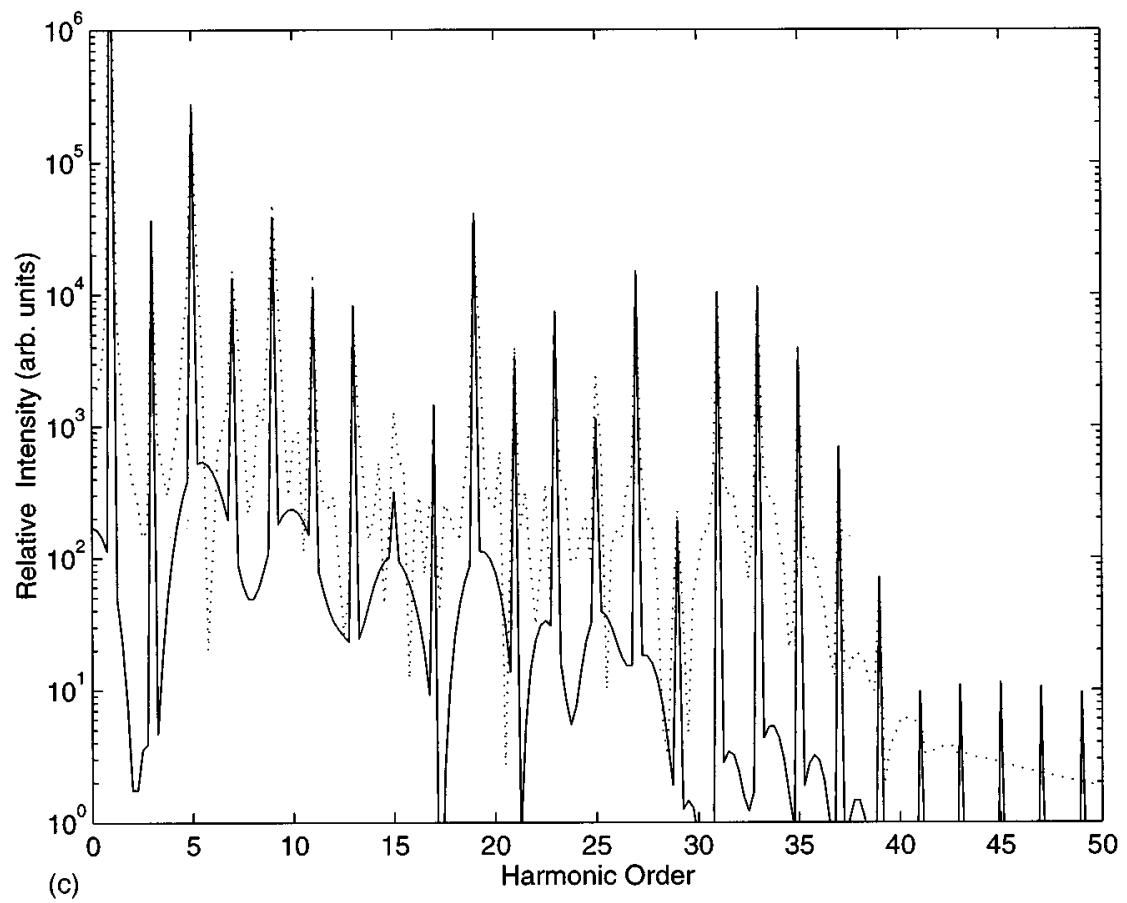

FIG. 3 (Continued).

harmonic response to an eight-cycle pulse of wavelength 372 $\mathrm{nm}$, with a field amplitude ramped linearly for intensities between $3.5 \times 10^{14}$ and $1.5 \times 10^{15} \mathrm{~W} / \mathrm{cm}^{2}$. The results are plotted in Fig. 4. In this case there is a clear blueshift of the highest harmonics, while the lower-order harmonics are still at the correct frequencies. At this stage the peaks are still clearly visible; however, there is a large amount of structure in between the harmonics. If we were to ramp the intensity faster, this structure would increase until it was no longer possible to see the harmonic peaks.

We will now pursue our theory further in order to study harmonic generation using such a time-dependent pulse. In previous sections we assumed that the behavior of each wavepacket was the same, and therefore we were able to write the dipole acceleration in the form of a convolution. For a time-dependent pulse we need to modify our approach to account for the fact that the behavior of a given wave packet will depend on the intensity of the field; i.e., each contribution will be different. The most straightforward method is to return to our single-cycle model, but rather than repeating an identical half-cycle, we build up the dipole acceleration from a series of half-cycles taken at different (constant) intensities.

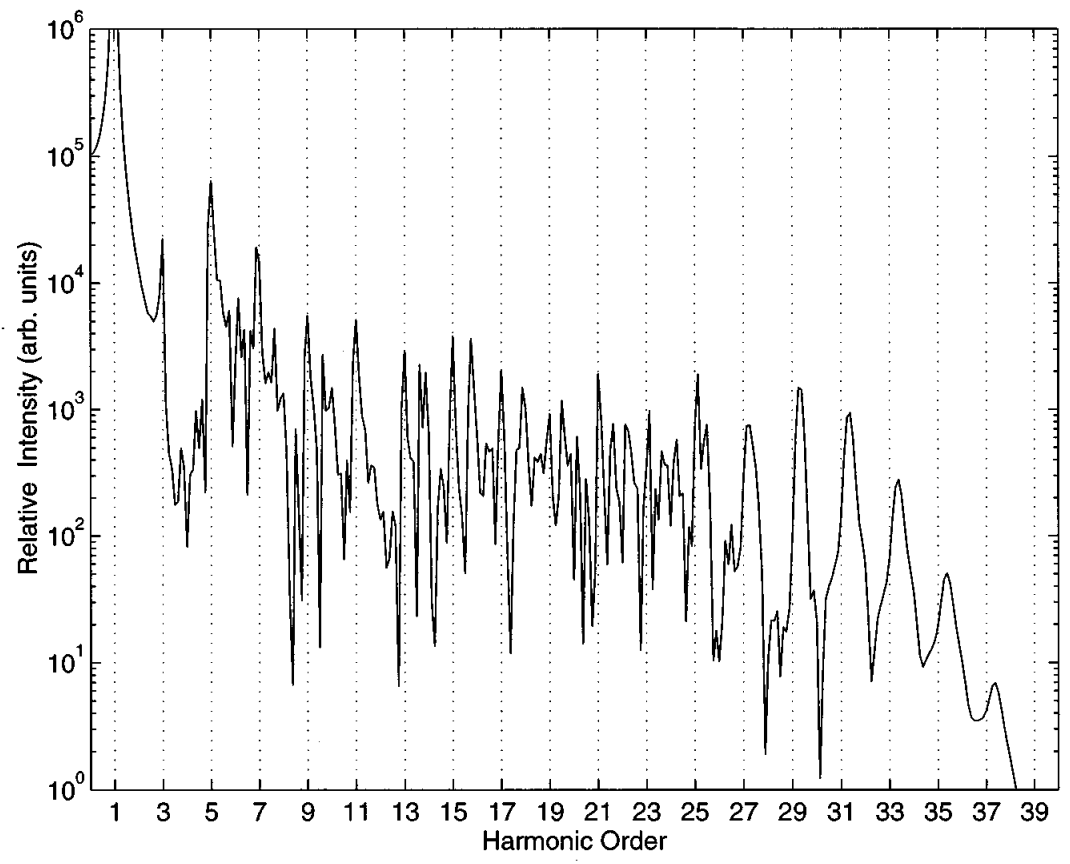

FIG. 4. The harmonic spectrum generated from $\mathrm{He}^{+}$using an eight-cycle pulse of wavelength $372 \mathrm{~nm}$, and peak intensity $1.5 \times 10^{15}$ $\mathrm{W} / \mathrm{cm}^{2}$, with the field ramped linearly over the pulse. 


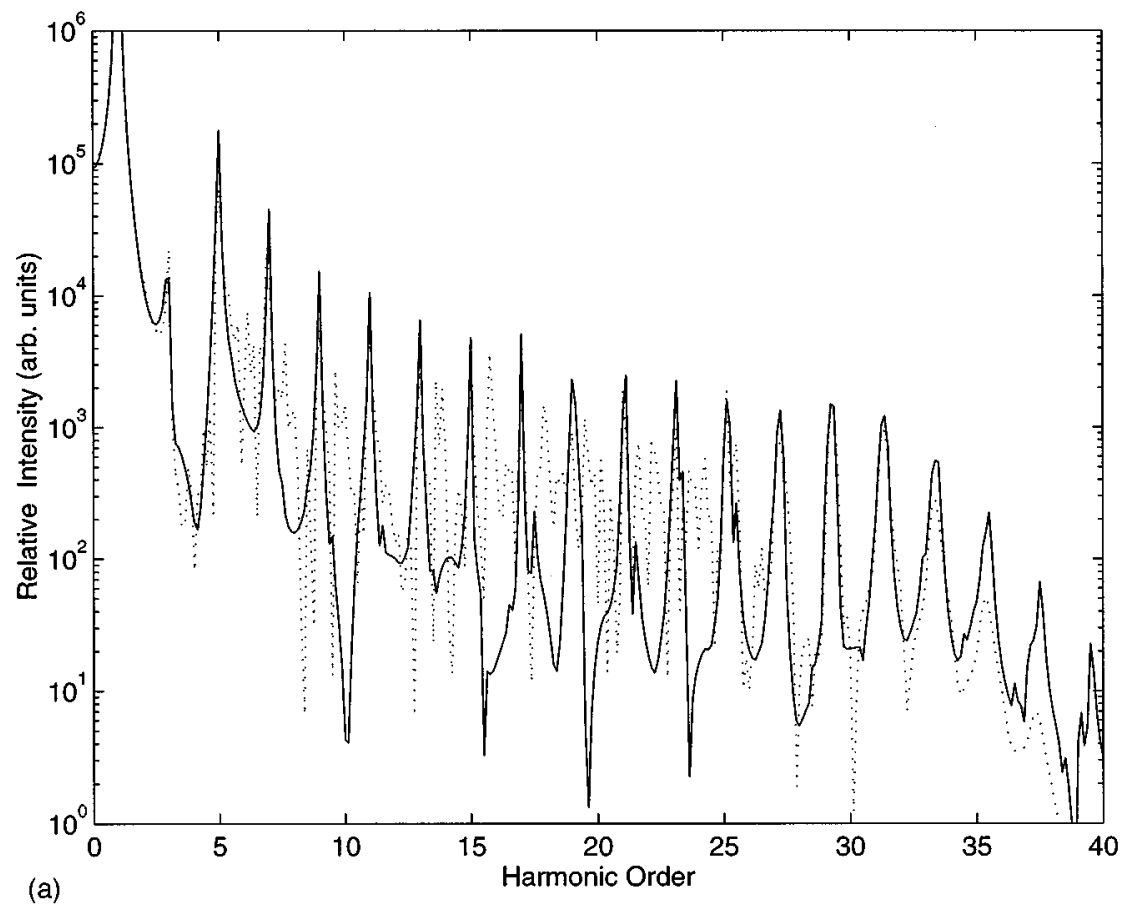

(a)

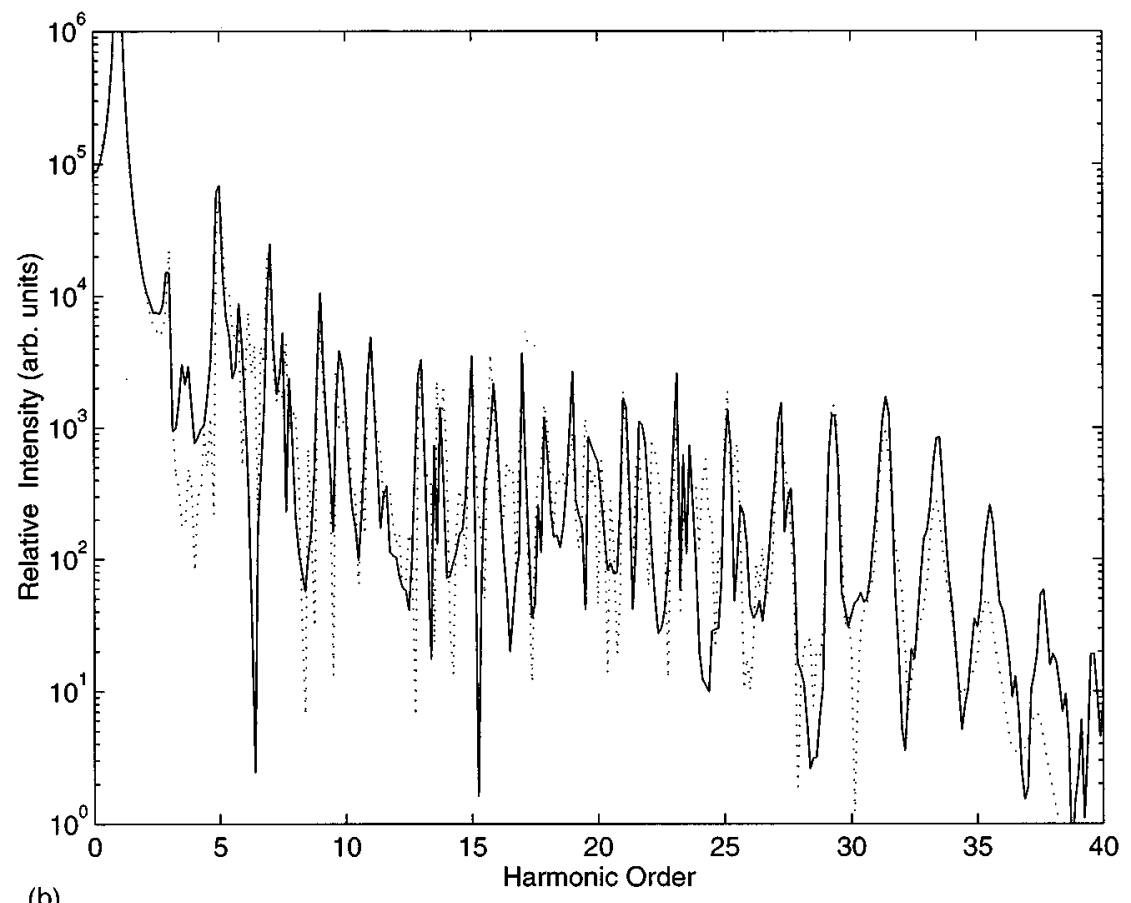

(b)

FIG. 5. The harmonic spectrum generated from $\mathrm{He}^{+}$using an eight-cycle pulse of wavelength $372 \mathrm{~nm}$, and peak intensity $1.5 \times 10^{15}$ $\mathrm{W} / \mathrm{cm}^{2}$, with the field ramped linearly over the pulse calculated by (a) using the first cycle of the response calculated at 16 different (constant) intensities, and (b) using the second cycle of the response calculated at 16 different (constant) intensities.

We propagated the TDSE for two laser cycles, at 16 different field amplitudes between 0.1 and 0.2 a.u. We then assume that the dipole acceleration for the full pulse is made up of the second half-cycle at each intensity in turn. The spectrum is plotted in Fig. 5(a) (to aid the comparison we have plotted the full spectrum as a dotted curve on the same plot). From the plot it is clear that the simple calculation perfectly replicates the position of the shifted "harmonic" peaks including the high-order, blueshifted peaks, but fails to generate any of the interharmonic structure.
By sampling the second half-cycle of the pulse at each intensity, we have removed effects due to multiple recollisions of a single wave packet. It is instructive to repeat the calculation, taking the acceleration from the second part of the second cycle at each intensity. In Fig. 5(b) we plot the result of this calculation; again we plot the full spectrum as a dotted curve. In this case there is much better agreement between the two cases. The calculation with multiple recollisions again matches the main peaks correctly, but this time 
we also obtain structure between the harmonics. If we combine this with the previous results we can fully explain the pulse-shape effects we observe. The first recollision (or more precisely the contribution within a single cycle) of each wave packet in turn leads to a set of very clean harmonic peaks, with no intermediate structure. The phase of each single recombination spectrum is dependent on the laser intensity, and therefore there is a time-dependent phase for each part of the spectrum, this causes a shift of the peaks in the spectrum away from the harmonic frequencies. We must also take into account the contribution to the spectrum due to a single wavepacket over several cycles, i.e., long trajectories and multiple recollisions. It is reasonable to assume that the longer a wave packet spends in the laser field, the greater the dependence of the phase on the laser intensity, and therefore the greater the blueshift. Thus the multiple contributions to a given part of the spectrum lead to structure in between the main peaks. In the case of a rapidly increasing field this structure becomes large, "smearing" the harmonic spectrum.

Our approach has therefore allowed us to explain both the single-atom blueshift, and also the loss of clear harmonic peaks. We can also make an important distinction between the effect of having different (average) intensities within different cycles of the pulse, with the fact that the intensity changes within a single cycle. If we think of this in terms of trajectories, then one might assume that the phase of the radiation produced during a single cycle would depend on both the average intensity during that cycle, and also on how the intensity changes within the cycle. Our results show that the change in the average laser intensity during different cycles leads to a shifting and smearing of the harmonic peaks, but the spectrum is not particularly sensitive to changes in intensity during a single cycle. While for calculations of the single-atom response this last point may appear trivial, it is important in calculations of the response of a collection of atoms. This collective response has previously been calculated by constructing a database, made up of the polarization (which is related to the dipole moment) of a single atom at a range of laser intensities. These data are then substituted into a wave equation to obtain the media response (see for example the work of Muffett [20]). Our conclusions here show that this approach may still be valid even when the laser intensity varies rapidly during the pulse.

We should point out that the results presented here are in qualitative agreement with the conclusions reached by Kan et al. [6] using both a semiclassical method, and also a method based on the formalism developed by Lewenstein et al. [5]. Their semiclassical method allowed them to study individual electron trajectories, and therefore they were able to make a much clearer distinction between the effect of the first collision and multiple collisions. In particular they found that only electrons undergoing their first recollision will have energies close to the cutoff; this explains why the cutoff harmonics are always well defined. For energies below the plateau they found that there are two possible trajectories (one either side of the main peak). The first of these trajectories leads to a peak close to the correct position, while the second has a large shift. Unfortunately the second of these electron trajectories does not return within the same cycle as it is produced; we are therefore unable to distinguish it from electrons that have returned more than once.

\section{CONCLUSION}

In this paper we have used a quantum-mechanical model to gain insight into the harmonic generation by considering separately the contribution due to the behavior of a single wave packet generated in the continuum, and the effect of periodic generation of such wave packets due to the periodicity of the driving field. We have demonstrated in the constant field amplitude limit that if we consider only the first recollision of each wave packet, we obtain a plateau of harmonics of equal intensity, followed by a cutoff in the expected position. If, however, we allow each wave packet to recollide more than once, and include the effect of wave packets that do not recollide within a single cycle, the harmonic plateau is modulated, with certain harmonics suppressed.

For a time-dependent pulse the first recollision of each wave packet once again generates a very clear series of peaks, although many of the peaks, particularly those close to the cutoff, are shifted from the harmonic frequencies. The shift is due to phase shifts between the spectra due to recollisions occurring at differing intensities. We have also found that recollisions that do not occur within a single cycle after the first cycle produce further peaks with a greater frequency shift. If the rate at which the field amplitude changes is sufficiently large, these shifts become comparable with the laser frequency, with the result that there are no clear peaks in the spectrum. The conclusions reached here have previously been presented by Kan et al. using a semiclassical approach based on the classical action. By using the formalism developed by Protopapas et al., we have been able to confirm their analysis using a fully quantum-mechanical analysis. Finally, we discussed the suitability of models based on the steadystate response for calculating the harmonic response in pulsed laser fields.

\section{ACKNOWLEDGMENTS}

We would like to acknowledge helpful discussions with C. H. Keitel, D. G. Lappas, M. Protopapas, and M. Lewenstein. The financial support of the European Community and of the U. K. Engineering and Physical Sciences Research Council is gratefully acknowledged.
[1] J. B. Watson, A. Sanpera, and K. Burnett, Phys. Rev. A 51, 1458 (1995).

[2] J. L. Krause, K. J. Schafer, and K. C. Kulander, Phys. Rev. Lett. 68, 3535 (1995).
[3] P. B. Corkum, Phys. Rev. Lett. 71, 1994 (1993).

[4] K. C. Kulander, K. J. Schafer, and J. L. Krause, Super Intense Laser-Atom Physics (SILAP III), edited by B. Piraux (Plenum, New York, 1993). 
[5] M. Lewenstein, Ph. Balcou, M. Yu. Ivanov, A. L'Huillier, and P. B. Corkum, Phys. Rev. A 49, 2117 (1994).

[6] C. Kan, C. E. Capjack, R. Rankin, and N. H. Burnett, Phys. Rev. A 52, R4336 (1995).

[7] M. Protopapas, D. G. Lappas, C. H. Keitel, and P. L. Knight, Phys. Rev. A 53, R2933 (1996).

[8] D. G. Lappas, M. V. Fedorov, and J. H. Eberly, Phys. Rev. A 47, 1327 (1993).

[9] F. I. Gauthey, C. H. Keitel, P. L. Knight, and A. Maquet, Phys. Rev. A 52, 525 (1995).

[10] R. V. Karapetyan and M. V. Fedorov, Zh. Éksp. Teor. Fiz. 75, 816 (1978) [Sov. Phys. JETP 48, 412 (1978)].

[11] P. Corkum, Opt. Photon. News, p. 18, (May 1995).

[12] See, for example, E. Hecht, Optics (Addison-Wesley, Reading, MA, 1987).
[13] G. D. Smith, Numerical Solution of Partial Differential Equations: Finite Difference Methods, 3rd ed. (Oxford University Press, Oxford, 1985).

[14] J. H. Eberly, R. Grobe, C. K. Law, and Q. Su, Atoms in Intense Laser Fields (Academic, New York, 1992).

[15] S. C. Rae, X. Chen, and K. Burnett, Phys. Rev. A 50, 1946 (1994).

[16] J. L. Krause, K. J. Schafer, and K. C. Kulander, Phys. Rev. A 45, 4998 (1992).

[17] F. Brunel, J. Opt. Soc. Am. B 7, 521 (1990).

[18] S. C. Rae and K. Burnett, J. Phys. B 26, 1509 (1993).

[19] A. Maquet, R. Taïeb, P. Antoine and B. Piraux (unpublished).

[20] Joanna Muffett, Ph.D. thesis, Imperial College, London, 1994; J. E. Muffett, C. G. Wahlstrom, and M. H. R. Hutchinson, J. Phys. B 27, 5693 (1994). 